This is an Author Accepted Manuscript of a conference paper presented at the Logistics Management and published by Springer Link. The definitive authenticated version is available online via http://dx.doi.org/10.1007/978-3-030-85843-8_5

Please use the following citation: Flechsig C. (2021) The Impact of Intelligent Process Automation on Purchasing and Supply Management - Initial Insights from a Multiple Case Study. In: Buscher U., Lasch R., Schönberger J. (eds) Logistics Management. Lecture Notes in Logistics. Springer, Cham. https://doi.org/10.1007/9783-030-85843-8 5

This research was supported by the EU project iDev40. The project iDev40 has received funding from the ECSEL Joint Undertaking (JU) under grant agreement No 783163. The JU receives support from the European Union's Horizon 2020 research and innovation programme. It is co-funded by the consortium members, grants from Austria, Germany, Belgium, Italy, Spain and Romania. The content of this paper does not reflect the official opinion of the Joint Undertaking ECSEL. Responsibility for the information and views expressed in the presentation lies entirely with the author.
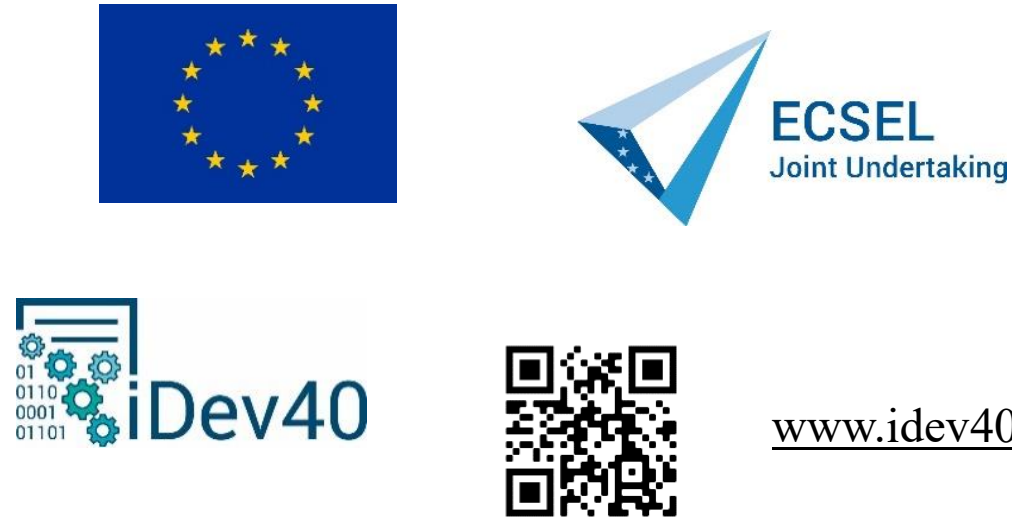

www.idev40.eu

SPONSORED BY THE

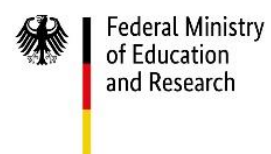

Diese Maßnahme wird mitfinanziert durch Steuermitte auf Grundlage des von den Abgeordneten des Sächsischen Landtags beschlossenen Haushaltes. 


\title{
The impact of Intelligent Process Automation on purchasing and supply management - Initial insights from a multiple case study
}

\author{
Christian Flechsig
}

Chair of Business Management, esp. Logistics Technische Universität Dresden, 01062 Dresden christian.flechsig@tu-dresden.de

\begin{abstract}
The Covid-19 pandemic drives the need for Intelligent Process Automation (IPA). However, the technology's adoption for purchasing and supply management (PSM) is still in the initial stage and has hardly been explored. Therefore, this empirical multiple case study builds on 19 organizations, including private and public procurement departments, consultancies, and IPA providers, to examine the impact of IPA on the PSM function. The findings provide comprehensive insights and reveal suitable operational and strategic application areas as well as several benefits related to IT systems and data, operational efficiency, process quality, and employee satisfaction. The study also identifies various technological, organizational, and environmental challenges that need to be overcome for further IPA adoption. Therefore, future research directions and managerial implications are outlined.
\end{abstract}

\section{Introduction}

The impact of the Covid-19 pandemic accelerates organizational change and the need for digital transformation and process automation (Coombs 2020). Due to its interface and networking function, the procurement department plays a vital role in Industry 4.0-related projects to enhance corporate performance and competitiveness (Bals et al. 2019). Buzz words like Procurement 4.0 (Bienhaus and Haddud 2018; Nicoletti 2020) outline the change of purchasing and supply management (PSM) towards a more strategically integrated business function. ${ }^{1}$ Nevertheless, the crucial digital transformation of PSM often lags behind other business functions (Allal-

\footnotetext{
${ }^{1}$ This paper uses the terms purchasing, procurement, and PSM interchangeably and refers to them as operational and strategic activities of private and public organizations "to ensure that the goods and services they need from their suppliers are available at the right time, in the right place, of the right quality, and at acceptable cost” (van Raaij 2016).
} 
Chérif et al. 2021; Hartley and Sawaya 2019). Although repetitive and low-valueadding processes based on structured data are increasingly being automated through e-procurement systems and the emerging Robotic Process Automation (RPA) technology, manual activities still dominate the purchasers' work routines. Most procurement tasks involve unstructured data, which challenges RPA and e-procurement systems (Nicoletti 2020; Schoenherr 2019). ${ }^{2}$

To overcome the shortcomings of RPA and enhance its capabilities and application areas, software providers increasingly integrate RPA with artificial intelligence (AI) (Mohanty and Vyas 2018; Syed et al. 2020). Following a recent leading AI conference's nomenclature, this paper refers to that advanced form as Intelligent Process Automation (IPA) (Chakraborti et al. 2020; Taulli 2020; Zhang 2019). IPA's market volume was valued at 10 billion USD in 2020 and is expected to grow with a compound annual growth rate of more than ten percent due to the impact of Industry 4.0 and the Covid-19 pandemic (Coombs 2020; Markets and Markets 2020). Along this line, procurement departments in the private sector consider intelligent technologies, like IPA, as core elements to make full use of their (unstructured) data and automate operational and strategic processes (Bienhaus and Haddud 2018; Jacobi and Groher 2019; Nicoletti 2020). IPA also receives growing interest from the public sector (Dias et al. 2019; Markets and Markets 2020). Despite the emerging practical dissemination, scientific research on IPA is still scarce (Ng et al. 2021; Syed et al. 2020; Viehhauser 2020), specifically in the context of PSM (Srai and Lorentz 2019). Existing publications (e.g., Allal-Chérif et al. 2021; Nicoletti 2020; Teli and Prasad 2018) are either generic or RPA-centered. They are also limited in their scope (i.e., focusing on benefits while neglecting challenges) and information base (e.g., interview studies involving private companies while neglecting public organizations). Therefore, PSM scholars and practitioners from private and public organizations are in great need of comprehensive and guiding research that examines the adoption of intelligent technologies and their potentials and challenges (Srai and Lorentz 2019; Van Hoek et al. 2020; Viale and Zouari 2020). To address this demand and the limitations of existing publications on IPA, this paper contributes to the following research questions (RQ):

RQ1: Which procurement processes are suitable for IPA?

RQ2: What are the potential benefits and challenges of IPA adoption?

RQ3: How does IPA influence the future development of PSM?

The paper follows a qualitative multiple case study approach to provide initial, yet comprehensive, empirical insights on IPA's impact on the PSM function. Nineteen semi-structured interviews with experts from private and public procurement organizations, consultancies, and software vendors were conducted. The study's

\footnotetext{
${ }^{2} \mathrm{RPA}$ is an umbrella term merging robotics and business process automation. Software licenses, so called "bots", mimic human behavior and automate "swivel chair work", i.e., repetitive, rulebased, and tedious business processes based on structured data. RPA is non-intelligent (Hofmann et al. 2020; Syed et al. 2020).
} 
contributions for researchers and practitioners are threefold: Firstly, a classification framework is developed to provide a common understanding of IPA. Secondly, potential application areas, benefits, challenges, and developments for procurement are presented. Lastly, the study points out future research directions and managerial implications to facilitate the further adoption of IPA in PSM. In that sense, the next chapter provides the relevant background. In chapter 3, the applied qualitative research methodology is described, while the results on the RQs are presented in chapter 4 and discussed in chapter 5 . The paper concludes with a summary and outlook.

\section{Intelligent Process Automation}

Hitherto, academia and practice lack a clear consensus on IPA terminology and components. Therefore, the following section provides a common understanding of IPA by explaining its essential components and characteristics. The proposed framework is also helpful to grasp the presented results in chapter 4. Besides, an overview of process automation in PSM is given in section 2.2.

\subsection{IPA classification framework}

IPA bots are considered a form of "weak $A \Gamma$ " as intelligence is applied only to specific areas. Bots are designed and trained for particular tasks, e.g., to convert data. In contrast to "strong $A F$ ", IPA bots cannot autonomously solve problems beyond their programmed parameters (Viehhauser 2020). ${ }^{3}$ The term IPA is also referred to as Cognitive Automation (e.g., Hofmann et al. 2020; Jacobi and Groher 2019; Taulli 2020; Willcocks 2020) or Intelligent Automation (e.g., Coombs 2020; $\mathrm{Ng}$ et al. 2021). However, it is reasonable to define IPA systems as the extension of basic, non-intelligent RPA with AI technologies. Therefore, IPA is part of Cognitive Automation, which in turn belongs to the broader concept of Cognitive Computing (Viehhauser 2020; Zhang 2019) (see Fig. 1).

\footnotetext{
${ }^{3}$ Usually, the term $A I$ is applied "when a machine mimics 'cognitive' and other functions that humans associate with human minds, for example, learning, problem solving, visioning, prediction and association" (Willcocks 2020).
} 


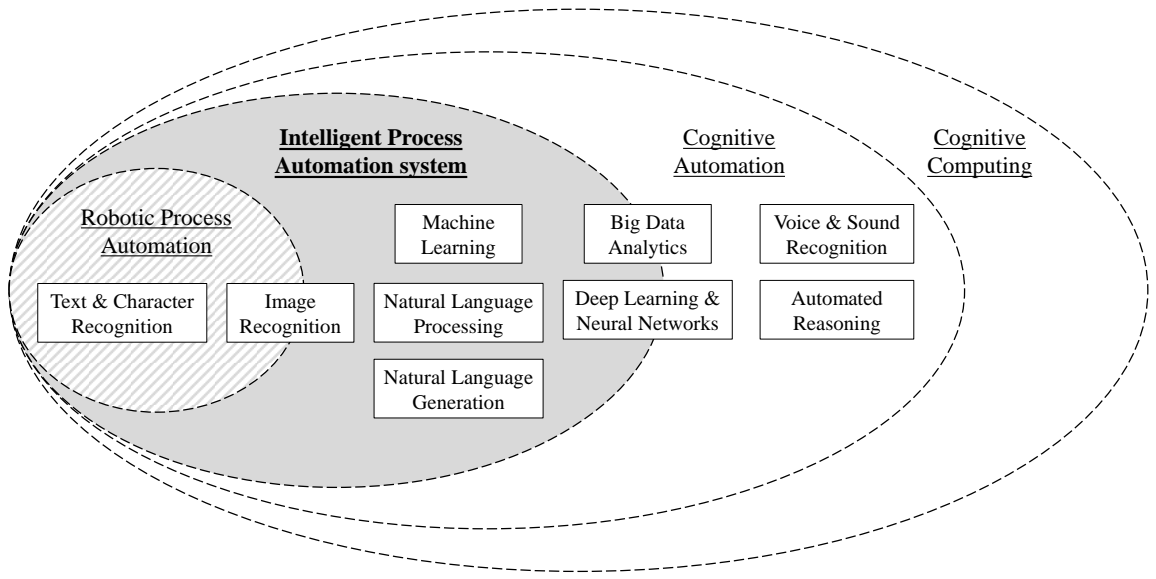

Fig. 1. IPA classification framework

Cognitive Computing is a subset of AI inspired by the human mind. It enables human-like interactions with users, interpretations and analyses of the contextual meanings, and autonomous decision-making based on deductions (Gupta et al. 2018). Cognitive Computing platforms comprise various advanced AI technologies, such as Machine Learning, Natural Language Processing, Natural Language Generation, Big Data Analytics, Deep Learning and Neural Networks, voice recognition, and automated reasoning (Nicoletti 2020; Viehhauser 2020). IPA systems extend RPA with several of those technologies to collect data and process information. Data is collected through text, character, and image recognition. While the first two are considered standard features of basic RPA, image recognition requires more sophisticated approaches, like Optical Character Recognition and Computer Vision (Mohanty and Vyas 2018; Viehhauser 2020). The former allows for converting unstructured to structured data, e.g., by extracting text from images, scans, or handwritten documents (Taulli 2020). Computer Vision draws on similarity analysis and visual conformance to identify, recognize, and categorize digital elements on user interfaces. Thus, the dependence on accessible underlying data is eliminated, enhancing the flexibility and application areas of bots (Viehhauser 2020).

For information processing, IPA relies on Machine Learning, Natural Language Processing, and Natural Language Generation (Mohanty and Vyas 2018; Zhang 2019). Machine Learning is an algorithm-based subset of AI for data pattern analyses (Hartley and Sawaya 2019). It is not explicitly programmed but learns and improves automatically to enable targeted predictions, recommendations, error handling, and responses to changing conditions (Mohanty and Vyas 2018; Taulli 2020). With IPA, Machine Learning typically refers to "supervised learning" as bots are trained with a large set of specific (historical) data to react to predefined output variables (Viehhauser 2020). Natural Language Processing enables the analysis and standardization of the unstructured oral or written input of conversations through 
contextual or sentiment analyses (Mohanty and Vyas 2018; Viehhauser 2020). Natural Language Generation is used to generate text or speech from that analyzed and structured information. IPA chatbots draw on both technologies to learn and communicate with customers and employees (Chakraborti et al. 2020; Gotthardt et al. 2020). Thus, they are applied for conversational processes based on unstructured data and human interaction ( $\mathrm{Ng}$ et al. 2021). In contrast to RPA, IPA chatbots (referred to as "virtual agents") are applied for both the back-office (i.e., employee services and supportive tasks) and the front-office (i.e., customer-facing businesses and interaction) (Allal-Chérif et al. 2021; Chakraborti et al. 2020; Mohanty and Vyas 2018).

While IPA and Cognitive Automation are both subsets of Cognitive Computing, Cognitive Automation goes beyond IPA as it iterates "its own automation approaches and algorithms for more expansive or more thorough analysis" (Suri et al. 2018). Thereby, Cognitive Automation uses more sophisticated Deep Learning and Big Data Analytics approaches based on cognitive domain knowledge (Taulli 2020; Zhang 2019). Although RPA and IPA are evolving towards Cognitive Automation (Hofmann et al. 2020), advanced cognitive capabilities, like voice or sound recognition and automated reasoning, are not incorporated yet (Viehhauser 2020).

In sum, IPA systems can be defined as platforms that integrate RPA with ERP systems and AI technologies through various interfaces to automate business processes that entail unstructured data and decision-making. Thereby, self-learning capabilities minimize the need for human interaction and training (Chakraborti et al. 2020: 219; Viehhauser 2020; Zhang 2019). In contrast, cognitive or intelligent RPA means that AI is directly incorporated into RPA itself and not added by external software via the platform approach (e.g., Syed et al. 2020; Teli and Prasad 2018; Viehhauser 2020).

\subsection{Process automation in purchasing and supply management}

Even though ERP and e-procurement systems are the backbones for procurement automation, they are often disparate and insufficient for real-time data exploitation, interoperability, and flexible decision-making (Hartley and Sawaya 2019; Schoenherr 2019). Therefore, organizations increasingly apply RPA as a bridge solution to automate manual, tedious, and rule-based processes while updating the essential IT systems within comprehensive projects (Jacobi and Groher 2019; Nicoletti 2020). Although scientific research on RPA for PSM is still nascent, initial studies show its positive impact, i.e., time and cost savings, employee reliefs, efficiency gains, error reduction, and increased quality of buyer-supplier relationships. RPA is applied in back-office processes to create purchase orders, support supplier communication, and collect and maintain data (Hartley and Sawaya 2019; Nicoletti 2020; Viale and Zouari 2020). However, RPA requires standardized workflows, exact programming, and structured data in a predefined format (Syed et al. 2020). 
Processing and converting the vast amount of unstructured data within procurement departments involves more sophisticated AI-based technologies (Allal-Chérif et al. 2021; Bienhaus and Haddud 2018). Therefore, IPA systems are an integral part of Procurement 4.0 (Nicoletti 2020; Schoenherr 2019). ${ }^{4}$

However, many organizations struggle with the required digital readiness (Bienhaus and Haddud 2018; Van Hoek et al. 2020). Thus, the adoption of advanced technologies in PSM is still nascent, particularly in small and medium-sized enterprises (SME) (Hartley and Sawaya 2019; Viale and Zouari 2020) and the public sector (Arlbjørn and Freytag 2012; Dias et al. 2019). Similarly, relevant scientific contributions for IPA adoption in procurement are limited. For example, Teli and Prasad (2018) emphasize the potentials of RPA and IPA (which they termed as Robotic Cognitive Automation). The publication examines several suitable application areas in supplier relationship and risk management, contract management, and master data management. However, the differentiation between RPA and IPA is not apparent. Since their interview study involved consultants only, the authors call for further empirical research covering different organizational levels and sectors. Hartley and Sawaya (2019) as well as Viale and Zouari (2020) focus on RPA benefits for procurement. They emphasize the necessity to implement more sophisticated approaches and investigate respective challenges for the PSM function. The urgent need to examine the impact of new automation technologies for procurement, including related application areas, benefits, and challenges, is also emphasized by other authors (Allal-Chérif et al. 2021; Jacobi and Groher 2019; Nicoletti 2020).

\section{Research methodology}

The qualitative multiple case study conducted to respond to that urgent research need contributes to the scarce body of scientific literature on IPA and generates initial yet rich insights on its impact on the PSM function. The qualitative multiple case study approach seems feasible as case study research is appropriate for in-depth investigations and analyses of contemporary phenomena within their real-world context to provide comprehensive insights and empirical descriptions for theory building (Eisenhardt and Graebner 2007; Yin 2018). Besides, case study research has already been applied for RPA and IPA (e.g., Hartley and Sawaya 2019; Viale and Zouari 2020; Viehhauser 2020). The research process followed the steps proposed by Stuart et al. (2002), i.e., defining research questions, developing the study design, selecting appropriate cases, collecting and analyzing the data.

\footnotetext{
${ }^{4}$ With Procurement 4.0, intelligent technologies process structured and unstructured data, execute natural human interactions, and draw deductions. They also automate strategic and recurrent planning processes like sourcing, supplier segmentation, negotiation, and contract management (AllalChérif et al 2021; Srai and Lorentz 2019).
} 


\subsection{Study design and case selection}

The study design draws on a blended inductive-deductive approach to develop new theory while also examining existing knowledge. The case selection is based on theoretical sampling to ensure external validity (Eisenhardt and Graebner 2007; Stuart et al. 2002). It was initiated by a broad search for private and public procurement organizations and facilitated by personal contacts to the "Association for Supply Chain Management, Procurement, and Logistics”, which comprises nearly 10,000 members in Central Europe and fosters the digital transformation of PSM. Suitable organizations for the multiple case study had to differ in their business fields and sizes and have planned, initial, or mature RPA and IPA applications.

In sum, 19 organizations were examined (see Table 1), involving six private and six public procurement departments. One polar case (Priv.F) was included among the private organizations to enrich the study's picture (Eisenhardt and Graebner 2007). Priv.F refrained from RPA implementation and recently rejected an IPA project. Besides, four digital procurement consultancies with extensive experience in RPA/IPA projects in both sectors were involved to yield more comprehensive data. Thereby, two consultancies (Cons.A, C) also provide self-developed RPA/IPA software. Additionally, three established RPA/IPA providers were included in the study, with Prov.A and Prov.B being global market leaders (Markets and Markets 2020). The respective sample sizes are consistent with the literature's proposals for qualitative case study research (Eisenhardt and Graebner 2007; Yin 2018). The participants are mainly headquartered in Germany and operate globally or throughout Europe.

\subsection{Data collection and analysis}

All participants received the research project's description and privacy statement beforehand to grant anonymity and build trust (Stuart et al. 2002). The respective informants were either directly contacted by the author or selected by the organization based on their job position, knowledge level, and willingness to participate in the study. Then, 19 semi-structured interviews were conducted, each consisting of up to three participating informants (i.e., 27 informants in total). Whenever possible, multiple informants per interview were preferred to diminish potential bias and enhance construct validity, as they often represented different viewpoints, hierarchical levels, and functional areas (Eisenhardt and Graebner 2007; Stuart et al. 2002). The semi-structured approach with open-ended questions (Yin 2018) allowed for flexibility and adjustments regarding the organization (type, level of RPA/IPA experience) and informants (knowledge, job position). All interviews took place in 2020 via telephone or face-to-face meetings and lasted 51 minutes on average. The 
informants had an average work experience of 19 years, with a minimum of four years and a maximum of 31 years.

Table 1. Anonymized overview of the 19 participating organizations

\begin{tabular}{|c|c|c|c|c|c|}
\hline Org. & Field & $\begin{array}{l}\text { Number of } \\
\text { employees }\end{array}$ & $\begin{array}{l}\text { Revenues } \\
\text { (in EUR M) }\end{array}$ & Positions of informants & $\begin{array}{l}\text { Dur. } \\
(\mathrm{min})\end{array}$ \\
\hline Priv.A & Automotive & $>50,000$ & $>1,000$ & IT Project Manager Purchasing & 25 \\
\hline Priv.B & Automotive & $>50,000$ & $>1,000$ & $\begin{array}{l}\text { Senior Purchasing Manager, } \\
\text { IT Project Manager Purchasing }\end{array}$ & 42 \\
\hline Priv.C & Automotive & $>50,000$ & $>1,000$ & IT Project Manager Purchasing & 50 \\
\hline Priv.D & Energy & $1,000-50,000$ & $>1,000$ & 2x Senior Purchasing Managers & 48 \\
\hline Priv.E & Engineering & $1,000-50,000$ & $>1,000$ & Senior Purchasing Manager & 29 \\
\hline Priv.F & Fashion & $1,000-50,000$ & $100-1,000$ & $\begin{array}{l}\text { Senior Purchasing Manager, } \\
\text { IT Project Manager Purchasing }\end{array}$ & 70 \\
\hline Publ.A & Energy & $1,000-50,000$ & $>1,000$ & $\begin{array}{l}\text { Senior Purchasing Manager, } \\
\text { Head of IT Competence Center, } \\
\text { IT Project Manager }\end{array}$ & 43 \\
\hline Publ.B & Finance & $1,000-50,000$ & $>1,000$ & Head of IT Competence Center & 58 \\
\hline Publ.C & Research & $1,000-50,000$ & $>1,000$ & Head of Purchasing & 51 \\
\hline Publ.D & Research & $1,000-50,000$ & $100-1,000$ & Senior Purchasing Manager & 49 \\
\hline Publ.E & Research & $1,000-50,000$ & $100-1,000$ & $\begin{array}{l}\text { Head of Purchasing, } \\
\text { Senior Purchasing Manager }\end{array}$ & 52 \\
\hline Publ.F & Research & $1,000-50,000$ & $100-1,000$ & $\begin{array}{l}\text { Head of Purchasing, } \\
\text { IT Project Manager Purchasing }\end{array}$ & 40 \\
\hline Cons.A & Procurement & $<1,000$ & $>1,000$ & Senior IT Consultant & 62 \\
\hline Cons.B & Procurement & $<1,000$ & $<100$ & Senior IT Consultant & 51 \\
\hline Cons.C & Procurement & $<1,000$ & $<100$ & Chief Executive Officer & 65 \\
\hline Cons.D & Procurement & $<1,000$ & $<100$ & Senior IT Consultant & 66 \\
\hline Prov.A & RPA/IPA & $1,000-50,000$ & $100-1,000$ & Head of Pre-sales & 68 \\
\hline Prov.B & RPA/IPA & $1,000-50,000$ & $100-1,000$ & 2x Senior IT Consultants & 60 \\
\hline Prov.C & RPA/IPA & $<1,000$ & $<100$ & Head of Research & 43 \\
\hline
\end{tabular}

Priv./Publ. $=$ Private/public procurement dept.; Cons. $=$ Consultancy; Prov. $=$ Software provider

Based on the research questions, two interview guidelines were developed and divided into four blocks: (1) opening questions about the organization and informant's experience; (2) understanding of terms and concepts (i.e., RPA, IPA, and PSM); (3) the impact of RPA and IPA on PSM, including application areas, benefits, challenges, and future developments; and (4) closing remarks. The first guideline was used for private and public organizations as well as consultancies (see Table 2). The second was applied to RPA/IPA providers due to their more holistic scope and included modified questions, for example, about integrated AI components and expectations for further technical development. While the interviews involved questions on both RPA and IPA, this paper focuses on the findings related 
to IPA. All interviews were recorded and transcribed verbatim. The transcripts were sent to the informants for review to ensure accuracy. Besides, supplementary material was included for data triangulation (Yin 2018), such as field notes, internal presentations, case documentations, client reports, product specifications, and the websites of the organizations.

Table 2. Excerpt of the first interview guideline with IPA-related questions

\begin{tabular}{ll}
\hline Category & Selected questions \\
\hline Application areas & $\begin{array}{l}\text { Which procurement tasks have you already automated/can/could you imagine } \\
\text { automating with IPA? Why? How are they characterized and prioritized? }\end{array}$ \\
Benefits & $\begin{array}{l}\text { What were/are/would be the motives for your organization to implement } \\
\text { IPA? Which benefits and capabilities of IPA did you realize/do/would you } \\
\text { expect? How is/would the performance impact (be) measured? }\end{array}$ \\
Challenges & $\begin{array}{l}\text { Which challenges and barriers did/do/would you face? How did/do/would } \\
\text { you cope with them? Which lessons did you learn from IPA/similar projects? }\end{array}$ \\
Outlook & $\begin{array}{l}\text { Which consequences and effects of large-scale IPA implementations do you } \\
\text { see/would you expect, with particular emphasis on PSM processes and em- } \\
\text { ployees? Which IPA-related issues should be addressed with future research? }\end{array}$ \\
\hline
\end{tabular}

The data analysis followed the principles of a systematic qualitative content analysis proposed by Mayring (2014) to ensure validity and reliability. The included data sources were iteratively coded with the software MAXQDA 2020. Firstly, the data were structured according to main categories, which were deductively elaborated from the interview guidelines and literature (e.g., the PSM process wheel of van Raaij (2016) to structure application areas, and the technology-organizationenvironment (TOE) framework of Tornatzky \& Fleischer (1990) to classify challenges). Secondly, a line-by-line text analysis was conducted to inductively develop related subcategories (e.g., specific IPA tasks, benefits, and challenges). Anchor samples were assigned to illustrate each category, and codes were grouped into themes. Then, the themes were contrasted, outlining similarities and differences. In the ultimate iteration, the final category system was applied to all collected data and emerged patterns were linked to relevant literature (Stuart et al. 2002). In addition, two peers were asked to code half of the interviews with the applied coding scheme to reduce bias, enhance reliability, and clarify discrepancies. The study ensures internal construct validity through a combined within-case and cross-case analysis to generate general impressions and cross-case patterns rather than individual insights (Mayring 2014; Yin 2018). 


\section{IPA in purchasing and supply management}

This chapter addresses the research questions by presenting the results on IPA's application areas, benefits, challenges, and impact on PSM development. The following section introduces the providers' perspective.

\subsection{The RPA/IPA software providers' perspective}

The providers' overall business goal is to offer an efficient and comprehensive software solution for business processes automation. Therefore, enhancing RPA with AI is regarded as a central objective. Depending on the providers' individual AI capabilities and strategies, considerable efforts are made to either incorporate AI components directly into RPA (Prov.A) and/or provide a functional and compatible IPA platform for third-party software integration (Prov.A, B, C). Although the IPA term differs among the providers due to unique selling propositions and marketing reasons (Prov.B), they concurred on the remaining of the umbrella term RPA as the basic and underlying technology. None of the examined RPA/IPA providers pursues a specialization strategy for individual branches or business functions, as Prov.A points out: "RPA [and IPA] is about accessing systems through user interfaces in the same way a human user would do, and that's completely universal."

Currently, the providers develop IPA systems by integrating RPA with the following AI components: Image recognition, ML, NLG, and NLP, with the last two being the primary focus as they enable the reading, processing, and transferring of unstructured data. IPA is usually more appropriate than RPA as it goes beyond operational processes and extends the application scope towards more strategic and value-adding tasks. Consequently, the providers stressed the high applicability and flexibility of IPA, i.e., there are, theoretically, almost no limits of use. However, they also emphasized that most AI technologies are still in an initial developmental stage and not yet applicable on a large scale. Therefore, the decision on how to supplement the IPA system with what AI feature lies with each customer and depends on the expected benefits (e.g., feasibility, profitability, and user value) and individual technological, organizational, and environmental conditions.

\subsection{Application areas}

IPA adoption is still at a very initial stage among the interviewed private and public procurement organizations. The consultancies and providers substantiated this observation. All informants agreed on RPA requiring AI upgrades to broaden its application areas. Thus, RPA experience was considered crucial for IPA 
implementation. Priv.A, B, C, D run mature RPA bots and already own initial IPA applications. In contrast, Priv.E and Publ.C, D, E, F are still on an early RPA level. Hence, they seek RPA experience before integrating AI features. Publ.A and Publ.B successfully finished initial RPA projects in the PSM function and currently pursue a broader roll-out and evaluation. Both organizations already plan the introduction of IPA. Priv.F refrained from RPA and IPA due to bad experiences and an already high degree of process automation through advanced e-procurement systems. However, the company currently prepares for the adoption of Machine Learning.

In sum, the informants named multiple suitable procurement tasks for IPA, which are structured according to the cyclical PSM process wheel of van Raaij (2016). The process wheel comprehensively visualizes the operational, tactical, and strategic activities of PSM (Bäckstrand et al. 2019). Thereby, the identification of the buying need, purchase order management, order fulfillment, invoice processing/approval, and payment are considered rather operational activities. Invoices provide inputs for more strategic and tactical procurement processes, such as sourcing analyses, need specification, sourcing strategy development, supplier selection, and contracting. Signed contracts initiate the next iteration of the PSM process wheel, i.e., buying need identification (van Raaij 2016). Table 3 responds to the first research question by summarizing procurement tasks for IPA. Many of them were mentioned by both private and public organizations. To the date of the interviews, none of the public participants had an IPA system in use. However, they expressed their ideas and plans for application areas of IPA in PSM, which mainly comprised strategic application areas related to need specification, tendering, and contracting.

According to the participants, intelligent bots are mainly applied in the backoffice to scan, digitize, standardize, capture, correlate, compare, and validate the vast amount of unstructured data (e.g., different supplier templates, structures, and units used for quotations, orders, and invoices). IPA's pattern recognition capabilities allow to predict demands and market trends, anticipate supply chain problems, initiate proactive approaches, and conduct in-depth spend analyses and supplier evaluations (Priv.B, C; Cons.A, C). IPA could support the extensive preparation of service descriptions for public organizations while ensuring compliance with the procurement law. In the subsequent tendering process, intelligent bots might recommend suitable tenderers and handle much of the communication (Publ.C, E).

In line with the efforts of RPA/IPA providers, the focus of recent IPA projects in the industry is the seamless interaction with internal (Priv.A, B, C, D) and external (Priv.C) stakeholders. Internally, chatbots are applied for "guided buying", i.e., they answer queries of human operators to generate targeted reports and lead the buyer to the ideal supplier for the desired product (Cons.A). In contrast, Priv.C also deploys IPA for "long-tail-optimizing" in the front-office to negotiate with suppliers for "C-Class purchases", i.e., non-recurring or low-frequent, non-strategic, indirect spends, which account for the minority of purchase volume but the majority of supplier and item numbers. Since Priv.C does not maintain an active relationship with those suppliers, the chatbot autonomously negotiates the contracts. Thereby, 
Machine Learning is used to define the optimal starting price as well as the maximal price for contract conclusion. Another bot then generates the purchase order.

Table 3. Suitable areas of IPA adoption in procurement, according to the PSM process wheel (van Raaij 2016)

\begin{tabular}{|c|c|}
\hline PSM process & Suitable tasks for IPA \\
\hline $\begin{array}{l}\text { Identification } \\
\text { of buying need }\end{array}$ & $\begin{array}{l}\text { Integrated category and inventory management (i.e., continuous real-time } \\
\text { monitoring and taking of inventory; joint forecasting and balancing of sup- } \\
\text { ply and demand; automatic replenishment; monitoring and prediction of } \\
\text { market trends; real-time e-catalog updates based on current inventory) }\end{array}$ \\
\hline $\begin{array}{l}\text { Purchase order } \\
\text { management }\end{array}$ & $\begin{array}{l}\text { Guided buying; generation, verification, categorization, and intelligent dis- } \\
\text { tribution of requests for quotation; digitization, standardization, and evalua- } \\
\text { tion of responses (e.g., format and data); optimized purchase order genera- } \\
\text { tion, approval, and sending to suitable suppliers based on real-time indices, } \\
\text { exchange rates, and raw material prices; purchase order updates }\end{array}$ \\
\hline Order fulfillment & $\begin{array}{l}\text { Planning and status tracking of consignments based on order numbers; } \\
\text { sending of notifications, alerts, and reminders to operators and suppliers; } \\
\text { comparison of the delivery note with the order; booking of incoming goods }\end{array}$ \\
\hline $\begin{array}{l}\text { Invoice processing } \\
\text { and approval }\end{array}$ & $\begin{array}{l}\text { Matching of request for quotation, purchase order, and invoice; checking, } \\
\text { processing, authorization (up to predefined limits), and posting of invoices; } \\
\text { digitization and standardization of various invoice formats }\end{array}$ \\
\hline Payment & Payment (up to predefined limits); posting of records and accounts payables \\
\hline Sourcing analyses & $\begin{array}{l}\text { Pattern recognition and classification of transactions with suppliers (spend } \\
\text { analyses); answering queries and generating reports for supplier evaluation } \\
\text { and risk management (e.g., scoring data to create risk profiles and potential } \\
\text { analyses); KPI tracking and benchmarking }\end{array}$ \\
\hline $\begin{array}{l}\text { Specification } \\
\text { of need }\end{array}$ & $\begin{array}{l}\text { Supply market research; product, price, and quality analyses; preparation of } \\
\text { service specifications; interpretation of procurement law }\end{array}$ \\
\hline $\begin{array}{l}\text { Sourcing strategy } \\
\text { development }\end{array}$ & $\begin{array}{l}\text { Standardization of templates for tendering; requesting information from po- } \\
\text { tential suppliers; regular internet searches for current information on suppli- } \\
\text { ers (e.g., negative press releases, difficulties, insolvency) }\end{array}$ \\
\hline Supplier selection & $\begin{array}{l}\text { Comparison of tenderer's responses; objective sourcing recommendations } \\
\text { based on supply market intelligence and analyses }\end{array}$ \\
\hline Contracting & $\begin{array}{l}\text { Supplier negotiation (e.g., optimized quantities and pricing), supplier } \\
\text { onboarding and communication (e.g., send evaluation forms, request (legal) } \\
\text { documents and quality certificates, check their validity and highlight dis- } \\
\text { crepancies, process and answer questions and complaints); reviewing, creat- } \\
\text { ing, and updating contracts and comparison to best-in-class templates; anal- } \\
\text { yses of contract usage to negotiate on adjustments, discounts, or penalties }\end{array}$ \\
\hline
\end{tabular}

\subsection{Benefits}

The informants concurred that IPA promises several benefits for PSM regarding IT systems and data, operational efficiency, process quality, and employee 
satisfaction. Table 4 responds to the first part of RQ2 by summarizing the participants' expected and experienced IPA benefits. Since most of the participants are yet on an early level with IPA, quantitative performance measures and concrete data are scarce. The consultancies and providers pointed out that further potentials might be discovered as many use cases still need to be explored, realized, and evaluated.

Table 4. Benefits of IPA adoption in procurement

\begin{tabular}{llll}
\hline IT systems and data & Operational efficiency & Process quality & Employee satisfaction \\
$\begin{array}{l}\text { Standardization of } \\
\text { unstructured data }\end{array}$ & $\begin{array}{l}\text { Fast data processing } \\
\text { and exchange }\end{array}$ & $\begin{array}{l}\text { Process optimization } \\
\text { and monitoring }\end{array}$ & $\begin{array}{l}\text { Workload reduction to } \\
\text { focus on cognitive tasks }\end{array}$ \\
Time savings & Objectivity & $\begin{array}{l}\text { Knowledge resource for } \\
\text { decision-making }\end{array}$ \\
$\begin{array}{l}\text { Scalability } \\
\text { Connectivity }\end{array}$ & $\begin{array}{l}\text { Productivity gains } \\
\text { Cost savings }\end{array}$ & Compliance & $\begin{array}{l}\text { Natural communication } \\
\text { Training and innovation }\end{array}$ \\
\hline
\end{tabular}

IT systems and data. IPA enables the processing and standardization of vast amounts of unstructured data and "hidden" information (Cons.C) in procurement (e.g., non-standardized oral and textual communication, non-machine-readable data like printed or scanned documents). Thereby, the technology enhances data quality and allows for flexible reactions to changing input and different supplier-dependent workflows and exceptions (Priv.C). Since bots are single software licenses, IPA platforms are scalable to the organization's current needs. Besides, they show high connectivity to many software applications and e-procurement systems and offset their shortcomings (Prov.A, B). Thus, IPA facilitates supplier integration and enables the complete automation of entire processes (Cons.C). An IT consultant of Prov.B summarized: "IPA is a platform to integrate future [AI] technologies into a business process without having to restructure the entire organization."

Operational efficiency. The high computing power of IPA systems allows for fast data processing and information exchange. Priv.C noticed substantial time savings. Since bots can autonomously operate day and night, they enhance efficiency and productivity and decrease the process cycle and idle times (Priv.A; Publ.A). Cons.B speculated that IPA, if well-integrated with the supply chain partners, could minimize supply chain inefficiencies that result from poor demand forecasts, unknown inventory, and delayed communication. Particularly private procurement organizations face much cost pressure and, therefore, apply intelligent bots to realize personnel savings (Priv.D; Cons.C). Priv.C implemented RPA and IPA to insource multiple processes formerly done by the shared service center and realized cost savings of around 50 external full-time equivalents. IPA constitutes an adequate alternative for traditional back-end automation depending on the application areas since IPA's return-on-investment (ROI) is usually higher (Cons.A, C, D).

Process quality. IPA facilitates the data-driven optimization, automation, and monitoring of procurement processes through pattern recognition and in-depth analyses. Bots provide objective results based on data instead of subjective feelings, 
thereby ensuring regulatory compliance (Publ.A; Cons.A). The informants agreed on a decreasing error rate as the bots' learning and experience levels increase, resulting in more appropriate behavior and accurate predictions. Priv.C reported that the quality of the automated results is at least as good as that of human operators.

Employee satisfaction. IPA frees up PSM professionals from tedious tasks and allows for a more valuable utilization of their skills and working time. Priv.A and Cons.C reported on workload savings of up to 60 percent. Thus, buyers can focus on activities that require human interaction and advanced cognitive capabilities, e.g., communication with essential suppliers and strategic sourcing decisions (Priv.B, D; Cons.D). Intelligent bots support decision-making with targeted analyses and objective recommendations (Cons.A). IPA can also store and pool the employees' knowledge (Cons.C). Priv.C, Publ.B, and Prov.B emphasized that the communication between a well-trained chatbot and humans proceeds naturally, which fosters its acceptance. Along this line, the informants agreed on higher satisfaction of employees and suppliers due to IPA, facilitating an innovative corporate culture and momentum for further automation. Affected employees are trained to handle AI systems and develop, maintain, monitor, and optimize bots (Priv.C, D; Publ.B). Cons.C reported: "[With IPA,] the know-how develops to higher levels."

\subsection{Challenges}

Multiple obstacles impede the nascent adoption of IPA in procurement. The participant's expected and experienced technological, organizational, and environmental challenges are summarized in Table 5, responding to the second part of RQ2. The classification follows the TOE framework of Tornatzky \& Fleischer (1990), which is a widely used approach to explain the adoption challenges of digital technologies (Kosmol et al. 2019).

Table 5. Challenges of IPA adoption in procurement, according to the TOE framework (Tornatzky and Fleischer 1990)

\begin{tabular}{lll}
\hline Technological challenges & Organizational challenges & Environmental challenges \\
\hline $\begin{array}{l}\text { Integration in the IT landscape } \\
\text { Requisite standardization and } \\
\text { optimization }\end{array}$ & $\begin{array}{l}\text { Governance } \\
\text { Internal communication and } \\
\text { collaboration }\end{array}$ & $\begin{array}{l}\text { Labor market availability } \\
\text { Diverse IPA platforms and pro- } \\
\text { viders }\end{array}$ \\
$\begin{array}{l}\text { Data collection and preparation } \\
\text { Training of AI algorithms }\end{array}$ & Lack of supportive culture & $\begin{array}{l}\text { Insufficient supplier readiness } \\
\text { Supply chain risks }\end{array}$ \\
$\begin{array}{l}\text { Monitoring and maintenance } \\
\text { Security issues }\end{array}$ & Lack of management support & Legal regulations \\
Lack of transparency & Lack of technical expertise & \\
Restricted capabilities & Deskilling & \\
\hline
\end{tabular}


Technological challenges. Many participants mentioned complex and disparate IT landscapes that impede the integration of new technologies. IPA challenges existing IT infrastructures as it requires more computing power than legacy ERP and e-procurement systems. Proper IT environments are needed to efficiently identify patterns, process data, and infer recommendations for decision-making (Prov.B, C). Intelligent bots require deep IT integration and usually receive their own user profiles and logins. Therefore, high data quality and reliability are crucial prerequisites for IPA since fragmented and disconnected information results in higher efforts for data collection and training (Priv.C). Thus, the comprehensive standardization and optimization of the IT, data, and process landscape before implementing IPA were key learnings of IPA adopters. The consultancies and providers reported on many procurement departments facing difficulties in providing a sufficient and comprehensive data pool on which IPA can be trained. That applies specifically to public organizations due to the high amount of non-recurrent purchases and frequently changing suppliers (Publ.A; Cons.D). Emphasizing the importance of the learning phase, the providers warned of excessive expectations since AI algorithms need a specific time to improve and learn how to separate relevant from irrelevant data.

Once deployed, intelligent bots require continuous monitoring and maintenance due to the higher complexity and more sensitive application than standard RPA (Priv.A). Priv.C and Publ.B pointed out that the proper programming of chatbots, reflecting human behavior as naturally as possible, is crucial for their acceptance and performance. The participants also emphasized the necessary security of data and IT systems. Hacker attacks and potential exposure of employees must be prevented, specifically, if IPA bots handle confidential and personal information (Priv.B; Cons.B). Manipulated training data could result in bias and severe consequences. Thus, IPA platforms need to be reliable, auditable, and scalable (Prov.B).

Although IPA increases data transparency, the underlying AI algorithms are considered "black boxes" and lack transparency (Priv.A). Despite considerable efforts undertaken by research and software providers, the still insufficient maturity of relevant AI components currently restricts IPA application areas to rather predictable and routine tasks (Priv.A, C; Cons.D). Besides, a zero-error rate is not yet realizable as the results are based on probabilities (Prov.B). Particularly public organizations raised concerns on IPA's applicability due to the complex nature of their procurement processes, e.g., the high number of indirect spend and human interactions (Publ.C, D, E). Priv.A pointed out: "The [IPA] algorithms are already good, but not mature yet. Currently, I wouldn't rely on the results."

Organizational challenges. Governance poses a critical issue for the trust and reliability of IPA. The informants found it challenging to establish control mechanisms, define clear responsibilities and rules, detail the human-bot communication, and determine the bot's scope of autonomy and decision-making. The importance of adequate communication and change management for IPA projects was strongly emphasized to yield intra-organizational synergies, prevent excessive expectations, and diminish user resistances (Priv.B; Publ.B). Besides, the issue of job loss anxiety 
should not be underestimated, particularly with intelligent bots capable of automating the more complex and creative tasks of employees (Cons.A, C).

The lack of supportive culture, specifically in public organizations, was also considered an impediment for IPA initiatives (Publ.C, F). Some participants experienced excessive bureaucracy and powerful yet obstinate works councils, resulting in high and tedious efforts for documentation, justification, and implementation (Priv.D; Publ.A; Cons.A). Therefore, rigid organizational structures need to become more agile, providing time and budget capacities to realize essential IT projects.

It was also reported on lacking strategies and readiness for the digital transformation of PSM (Cons.B; Publ.D). Thus, IPA adoption suffers from poor sponsorship and guidance of the top management. Furthermore, skills required for Procurement 4.0 (e.g., technical and analytical capabilities, holistic and open-minded thinking) are yet to be developed through training (Priv.E, F; Cons.A). In contrast, the concern of potential deskilling was raised (Cons.D). With IPA, employees could be tempted to rely excessively on the bot's recommendations instead of using their minds. Therefore, professionals need further training to evaluate IPA's outcome critically and to prevent the loss of their creative and judgment capabilities.

Considerable implementation costs constitute another crucial adoption barrier (Priv.E, F). The ROI of IPA is much lower than for RPA due to the more complicated and deeper IT integration, higher initial investments, and license costs (Cons.A, C; Prov.A). That also includes higher costs for bot onboarding and maintenance. Cons.C and Prov.C speculated that those expenses could impede the adoption at SMEs. The polar case organization Priv.F terminated an initial IPA project due to the high costs for implementation and training as well as lacking capabilities and performance of IPA. Priv.E explained: "With RPA, we're talking about 50,000 Euros per process. With AI, we're talking about a factor of ten."

Environmental challenges. Since most interviewed organizations seek the implementation and upscaling of AI technologies, qualified experts for such projects pose a scarce resource. Due to the rigid hierarchies and salary structures, public organizations struggle to find skilled employees (Publ.C, D, E, F). Another challenge is the growing variety of IPA providers and platforms, each with different functionalities and license models. Therefore, the crucial in-depth analysis of suitable partners and systems is very time-consuming (Priv.A, C; Cons.B). AI also dehumanizes buyer-supplier relationships (Priv.C). While Priv.C and Cons.C stated that the suppliers' collaboration and digital readiness are neglectable for IPA, their importance was strongly emphasized by Cons. A, B, D since lacking supplier readiness impedes the adoption of new technologies within the supply chain. Faulty and untuned bots could cause severe consequences, e.g., distribution delays and supply shortages due to incorrect purchase orders or payments.

Legal regulations, specifically national and transnational procurement law, constitute a substantial challenge for public organizations. They do not necessarily maintain strong buyer-supplier relationships due to the mandatory objective tendering of most purchases. Therefore, the restrictive rules could impede an optimization mindset and limit the scope for action (Publ.B, C, E). Thus, bots need continuous 
and extensive training on interpreting different legal regulations to ensure high compliance when they are applied in public tendering processes. Cons.A summarized: "Process automation is always a sensitive topic, especially in the public sector."

\subsection{Impact on the future development of procurement}

Providing implications for future research and responding to the third research question, the mentioned impacts of IPA adoption on the future development of procurement can be classified into three major issues: the technology, PSM function, and PSM employees.

Technology. The participants concurred on the importance of the flexible IPA platform approach and profound RPA experience for successful projects. RPA will remain cheap and more applicable for specific rule-based and structured procurement tasks. Therefore, IPA and RPA often go hand in hand (Priv.A; Publ.B; Cons.C; Prov.B): IPA can structure and standardize data before passing to an RPA bot. In contrast, AI technologies can be applied to the RPA output to conduct more in-depth analyses, pattern recognition, predictions, and decision-making (Priv.C; Cons.A). Due to the high complexity of IPA, the consultancies and providers recommended organizations not to develop their own solutions and reported on negative experiences of respective customers. Indeed, all IPA adopters rely on external software platforms and support. However, building internal know-how to develop, deploy, and maintain intelligent bots was strongly emphasized and regarded as a vital prerequisite for further adoption (Priv.A, C; Publ.A, B). Besides, voice recognition and deep learning are highly desired capabilities (Priv.D; Cons.C, D). Although most participants considered AI components crucial for achieving a high degree of process automation in procurement, the experts of the polar case organization Priv.F stated: "AI would have only made sense if we had pursued a large-scale implementation to save people. But that wasn't the case."

PSM function. Many interviewed purchasing managers drive their organizations' digital transformation. The informants concurred that procurement departments need to become more agile and provide a sufficient data pool for IPA training by restructuring and standardizing their workflows. Besides, highly digitized departments within the same enterprise, innovative start-up companies or suppliers, and specialized fairs provide suitable possibilities for mutual exchange and learning (Priv.B; Publ.B). All informants agreed on IPA accelerating the ongoing change of the PSM function towards an increased strategic focus. Operational activities will be decreasing and performed by bots and other intelligent systems. In return, the communication and quality of internal and external relationships will become more relevant, e.g., due to supplier integration. Cons.B claimed: "In my opinion, operational purchasing and buyers will definitely disappear."

PSM employees. Although process automation naturally evokes employee anxieties, the informants reported that most PSM professionals are open-minded 
towards IPA and give higher weight to the resulting workload relief than corresponding fears and reservations. In light of the upcoming demographical change, digital transformation and automation are vital to enhance the organization's attractiveness and recruit necessary experts (Priv.D, E; Publ.A, C, D). However, IPA adoption should go hand in hand with holistic change management, involving affected employees as future buyers need an adjusted skill set, e.g., profound abilities related to IT, automation, analyses, communication, and strategic thinking (Cons.B, C). Therefore, procurement departments must undertake high training, coaching, and reskilling efforts, resulting in new job profiles (Priv.A, C; Cons.A) and potentially higher salary grouping of the employees (Publ.D, E). Priv.C and Publ.B emphasized the allocation of responsibilities to facilitate seamless human-bot collaboration. Finally, the experts of Priv.D disagreed with Cons.B's statement: “We don't think that an operational buyer is completely replaceable with AI."

\section{Discussion}

Since this study is among the first that qualitatively examines IPA adoption in procurement, it provides initial yet profound insights into suitable application areas, related benefits and challenges, and IPA's impact on the future development of the PSM discipline. In the following, the findings are discussed with relevant literature to outline theoretical and managerial implications. Thereby, future research directions and practical recommendations are deduced to facilitate the still-nascent adoption of IPA in procurement. Moreover, the study's limitations are explained.

\subsection{Theoretical implications}

The results outline the increasing impact of IPA on the PSM function and indicate its value potentials, thereby substantiating and enriching the rather general findings of the limited IPA research in purchasing (Allal-Chérif et al. 2021; Nicoletti 2020; Teli and Prasad 2018). It was found that most of the discovered benefits and challenges of IPA for PSM also apply to other business functions like accounting or auditing (Gotthardt et al. 2020; Zhang 2019).

This study complements and extends prior work by presenting novel application areas and emphasizing previously neglected aspects of IPA, such as IT integration, restricted capabilities, lack of supportive culture and technical expertise, implementation costs, legal regulations, and supplier issues. However, the findings also deviate from the literature in specific points. Thereby, aspects that might not be mentioned do not necessarily imply irrelevance but indicate that other factors might be more relevant to the participants. In the study, for example, the cost-saving potentials of IPA were predominantly related to personnel savings. Beyond that, IPA also 
decreases the costs for transactions and maverick buying due to a higher spending control and improved sourcing (Allal-Chérif et al. 2021; Nicoletti 2020). Furthermore, the experts highlighted substantial training efforts of employees, while IPA can also decrease the training need on legacy e-procurement systems and applications (Nicoletti 2020). Most informants neglected the "black box problem" of IPA's underlying AI algorithms. However, this essential issue should be addressed by developing transparent and interpretable models (Gotthardt et al. 2020).

The participants require more detailed guidance for IPA implementation in PSM and transition from RPA than currently provided by the literature. Therefore, corresponding to RQ3, future conceptional and empirical research should build on the technical, organizational, and environmental challenges identified in this study and provide answers to urgent questions of the informants, such as: How to implement and upscale RPA and IPA in PSM? Which platforms, license models, and sourcing options are appropriate for which contexts? How to build know-how and realize the knowledge transfer from private to public procurement? How to identify and select suitable processes and measure the performance impact? How to increase the transparency of IPA procedures? When to trust a bot and allow for autonomous decisions? What type of human-bot or bot-bot collaboration is appropriate, and which capabilities of humans and bots are required? How to develop and communicate an appropriate digital transformation strategy for PSM? How to facilitate IPA adoption on both sides of the buyer-supplier dyad? How to establish and maintain good buyer-supplier relationships with IPA? How to attract and retain qualified experts with IT and AI skills for procurement departments, particularly in the public sector?

\subsection{Managerial implications}

The study findings can serve as reference points for managers in the private and public sector to facilitate IPA adoption in procurement and identify initial application areas. While the technology promises high potentials, the results indicate that the related technological, organizational, and environmental challenges should be tackled first. The often lacking digital readiness of procurement departments impedes IPA adoption, particularly in the public sector (Hartley and Sawaya 2019; Kosmol et al. 2019). However, as a central and vital business function, purchasing needs to foster the digital transformation and become more internally integrated and strategically focused since isolated AI initiatives quickly fizzle out (Allal-Chérif et al. 2021; Bienhaus and Haddud 2018). Besides, AI dehumanizes buyer-supplier relationships (Allal-Chérif et al. 2021; Bals et al. 2019; Kosmol et al. 2019). While the positive and forward-looking attitude of the participants' employees reflects the prevailing scientific discourse (e.g., Van Looy 2020; Willcocks 2020; Zhang 2019), low-skilled workers could find it challenging to compete with intelligent bots and, thus, resist the fundamental digital transformation. Therefore, procurement department managers should consider the changing skill sets and job profiles of future 
buyers and increase the awareness for Procurement 4.0 among their employees and supply chain partners. In addition, the top-level management has to enhance digital readiness by developing a targeted PSM digitalization strategy that includes IPA.

Although the providers stressed the high applicability and flexibility of IPA, intelligent bots are still restricted to rather repetitive, non-creative, and notknowledge-intensive processes. Workflows that require contextual decisions or social activities based on human interaction and empathy (e.g., for motivating or convincing) are currently less suitable for IPA (Van Looy 2020). Therefore, software providers need to enhance the capabilities. At the same time, organizations should provide the prerequisites for IPA adoption by standardizing complex IT landscapes and workflows and ensuring high data quality and reliability.

Before introducing the technology, organizations should conduct profound costbenefit calculations and provider analyses since the high implementation costs pose a considerable challenge (Chakraborti et al. 2020; Gotthardt et al. 2020; Zhang 2019). Therefore, RPA can be a cheap and sufficient alternative for specific tasks and an initial step for large-scale process automation. However, training efforts for employees as well as the comprehensive and reliable data pools required for the efficient learning of intelligent bots, should not be underestimated. Therefore, holistic change management guides the adoption of IPA, dissipating employee resistances and preventing excessive expectations.

Furthermore, decision-makers should expand the scope of IPA projects on other departments and the supply network to drive joint initiatives. The co-evolution of the buyer-supplier dyad fosters the digital readiness and implementation of intelligent technologies for procurement (Kosmol et al. 2019). When preparing IPA projects and bot deployment, particularly public organizations need to consider procurement law and legal regulations regarding AI integration. Finally, organizations should invest in enhancing their attractiveness to recruit and retain qualified experts early on.

\subsection{Limitations of the study}

Despite the applied methodological rigor, this empirical paper is subject to some limitations that need to be considered when interpreting the findings.

Firstly, as a qualitative interview study, the number and variety of the examined organizations are restricted. Although the chosen approach yielded rich primary and secondary data, particularly from providers and consultancies (e.g., client reports), the results mainly build on large organizations headquartered in Europe. However, IPA adoption is expected to grow also in emerging countries and SMEs (Markets and Markets 2020). Thus, future studies should involve organizations from different geographical locations (e.g., North America, Asia), branches, and sizes.

Secondly, although the inclusion of informants from various hierarchical levels and domains enriched the debate, interviews with more than one expert could suffer 
from a potential bias as informants might not speak freely or could be tempted to just agree with another opinion. Therefore, more objective, quantitative approaches could confirm, complement, or disprove the presented findings.

Finally, IPA adoption is still in its infancy in most examined procurement organizations, particularly in public ones. Therefore, this study seeks to provide practitioners and scholars with initial empirical insights on application areas, benefits, and challenges to successfully start and conduct an IPA initiative. However, future research should go beyond and also tackle issues related to the upscaling of IPA and AI technologies that still lack profound practical experiences and theoretical underpinning (Chakraborti et al. 2020; Gotthardt et al. 2020). For example, potential synergies and interrelations with other business functions and suppliers could be investigated.

\section{Conclusion}

This pioneering multiple case study contributes to the nascent body of literature by providing comprehensive insights on the PSM impact of the rapidly disseminating IPA technology. Nineteen organizations were interviewed, including private and public procurement departments, procurement consultancies, and RPA/IPA software providers. The implications of this paper could provide a starting point for further research and practical adoption. IPA facilitates the saving of time and costs as well as thorough decision-making, prediction, data gathering, preparation, and analysis. Thereby, the technology is applicable for multiple operational, tactical, and strategic procurement tasks. Organizations benefit from the increasing level of standardization, operational efficiency, process quality, and employee satisfaction. However, for more widespread adoption in PSM and other business functions, IPA needs to overcome various technological, organizational, and environmental challenges detected by this study. The quote of an IT project manager of Priv.B summarizes the paper's essence: “IPA has much potential and will definitely emerge. The adoption is just a matter of organizational readiness and courage. However, the movie "Terminator" used to amuse me, but now it just scares me."

\footnotetext{
Acknowledgments This research was supported by the EU project iDev40. The project iDev40 has received funding from the ECSEL Joint Undertaking (JU) under grant agreement No 783163. The JU receives support from the European Union's Horizon 2020 research and innovation programme. It is co-funded by the consortium members, grants from Austria, Germany, Belgium, Italy, Spain, and Romania. The content of this article does not reflect the official opinion of the Joint Undertaking ECSEL. Responsibility for the information and views expressed in the article lies entirely with the author. In addition, the author would like to express his sincere gratitude to Lena Otto, who has contributed to the improvement of earlier versions of this paper.
} 


\section{References}

Allal-Chérif O, Simón-Moya V, Ballester ACC (2021) Intelligent purchasing: How artificial intelligence can redefine the purchasing function. J. Bus. Res. 124:69-76. https://doi.org/10.1016/j.jbusres.2020.11.050

Arlbjørn JS, Freytag PV (2012) Public procurement vs private purchasing: Is there any foundation for comparing and learning across the sectors? Int. J. Public Sec. Manag. 25:203-220. https://doi.org/10.1108/09513551211226539

Bäckstrand J, Suurmond R, van Raaij E, Chen C (2019) Purchasing process models: Inspiration for teaching purchasing and supply management. J. Purch. Supply Manag. 25:100577. https://doi.org/10.1016/j.pursup.2019.100577

Bals L, Schulze H, Kelly S, Stek K (2019) Purchasing and supply management (PSM) competencies: Current and future requirements. J. Purch. Supply Manag. 25:100572. https://doi.org/10.1016/j.pursup.2019.100572

Bienhaus F, Haddud A (2018) Procurement 4.0: factors influencing the digitisation of procurement and supply chains. Bus. Process Manag. J. 24:965-984. https://doi.org/10.1108/BPMJ-062017-0139

Chakraborti T, Isahagian V, Khalaf R, Khazaeni Y (2020) From Robotic Process Automation to Intelligent Process Automation - Emerging Trends. In: Asatiani A, García JM, Helander N, Jiménez-Ramírez A (eds) Business Process Management: Blockchain and Robotic Process Automation Forum. Springer International Publishing, Cham, pp 215-228

Coombs C (2020) Will COVID-19 be the tipping point for the Intelligent Automation of work? A review of the debate and implications for research. Int. J. Inf. Manag. 55:102182. https://doi.org/10.1016/j.ijinfomgt.2020.102182

Dias M, Pan S, Tim Y (2019) Knowledge Embodiment of Human and Machine Interactions: Robotic Process Automation at the Finland Government. In: Proceedings of the 27th European Conference on Information Systems

Eisenhardt KM, Graebner ME (2007) Theory Building From Cases: Opportunities And Challenges. AMJ 50:25-32. https://doi.org/10.5465/amj.2007.24160888

Gotthardt M, Koivulaakso D, Paksoy O, Saramo C, Martikainen M, Lehner O (2020) Current State and Challenges in the Implementation of Smart Robotic Process Automation in Accounting and Auditing. J. Finance Risk Perspect. 9:90-102. https://doi.org/10.35944/jofrp.2020.9.1.007

Gupta S, Kar AK, Baabdullah A, Al-Khowaiter WA (2018) Big data with cognitive computing: A review for the future. Int. J. Inf. Manag. 42:78-89. https://doi.org/10.1016/j.ijinfomgt.2018.06.005

Hartley JL, Sawaya WJ (2019) Tortoise, not the hare: Digital transformation of supply chain business processes. Bus. Horiz. 62:707-715. https://doi.org/10.1016/j.bushor.2019.07.006

Hofmann P, Samp C, Urbach N (2020) Robotic process automation. Electron. Mark. 30:99-106. https://doi.org/10.1007/s12525-019-00365-8

Jacobi C, Groher E (2019) Beschaffung 4.0. In: Schröder M, Wegner K (eds) Logistik im Wandel der Zeit - Von der Produktionssteuerung zu vernetzten Supply Chains. SpringerGabler, Wiesbaden, pp 545-566

Kosmol T, Reimann F, Kaufmann L (2019) You'll never walk alone: Why we need a supply chain practice view on digital procurement. J. Purch. Supply Manag. 25:1-17. https://doi.org/10.1016/j.pursup.2019.100553

Markets and Markets (2020) COVID-19 Impact on Intelligent Process Automation Market by Component, Vertical, and Region - Global Forecast to 2025. https://www.marketsandmarkets.com/Market-Reports/covid-19-impact-on-intelligent-process-automation-market39284189.html. Accessed 28 February 2021

Mayring P (2014) Qualitative content analysis: theoretical foundation, basic procedures and software solution, Klagenfurt 
Mohanty S, Vyas S (2018) How to Compete in the Age of Artificial Intelligence. Apress L.P, Berkeley, CA

$\mathrm{Ng} \mathrm{KK}$, Chen C-H, Lee C, Jiao J, Yang Z-X (2021) A systematic literature review on intelligent automation: Aligning concepts from theory, practice, and future perspectives. Adv. Eng. Inform. 47:101246. https://doi.org/10.1016/j.aei.2021.101246

Nicoletti B (2020) Procurement 4.0 and the Fourth Industrial Revolution: The Opportunities and Challenges of a Digital World. Palgrave Macmillan, Cham

Schoenherr T (2019) The Evolution of Electronic Procurement. Springer International Publishing, Cham

Srai JS, Lorentz H (2019) Developing design principles for the digitalisation of purchasing and supply management. J. Purch. Supply Manag. 25:78-98. https://doi.org/10.1016/j.pursup.2018.07.001

Stuart I, McCutcheon D, Handfield R, McLachlin R, Samson D (2002) Effective case research in operations management: a process perspective. J. Oper. Manag. 20:419-433. https://doi.org/10.1016/S0272-6963(02)00022-0

Suri VK, Elia MD, Arora P, van Hillegersberg J (2018) Automation of Knowledge-Based Shared Services and Centers of Expertise. In: Kotlarsky J, Oshri I, Willcocks L (eds) Digital Services and Platforms. Considerations for Sourcing. Springer International Publishing, Cham, pp 5675

Syed R, Suriadi S, Adams M, Bandara W, Leemans SJJ, Ouyang C, ter Hofstede AH, van de Weerd I, Wynn MT, Reijers HA (2020) Robotic Process Automation: Contemporary themes and challenges. Comput. Ind. 115:103162. https://doi.org/10.1016/j.compind.2019.103162

Taulli T (2020) The Robotic Process Automation Handbook. Apress, Berkeley, CA

Teli R, Prasad SK (2018) Delivering Value in Procurement With Robotic Cognitive Automation (RCA) Services. Int. J. Robot. Appl. Technol. 6:1-11. https://doi.org/10.4018/IJRAT.2018070101

Tornatzky LG, Fleischer M (1990) The processes of technological innovation. Issues in organization and management series. Lexington Books, Lexington

Van Hoek R, Sankararaman V, Udesen T, Geurts T, Palumbo-Miele D (2020) Where we are heading and the research that can help us get there - Executive perspectives on the anniversary of the Journal of Purchasing and Supply Management. J. Purch. Supply Manag. 26:100621. https://doi.org/10.1016/j.pursup.2020.100621

Van Looy A (2020) Adding Intelligent Robots to Business Processes: A Dilemma Analysis of Employees' Attitudes. In: Fahland D, Ghidini C, Becker J, Dumas M (eds) Business Process Management. Springer International Publishing, Cham, pp 435-452

van Raaij E (2016) Purchasing value: Purchasing and supply management's contribution to health service performance. Erasmus Reseach Institute of Management, Rotterdam

Viale L, Zouari D (2020) Impact of digitalization on procurement: the case of robotic process automation. Supply Chain Forum: Int. J. 21:185-195. https://doi.org/10.1080/16258312.2020.1776089

Viehhauser J (2020) Is Robotic Process Automation Becoming Intelligent? Early Evidence of Influences of Artificial Intelligence on Robotic Process Automation. In: Asatiani A, García JM, Helander N, Jiménez-Ramírez A (eds) Business Process Management: Blockchain and Robotic Process Automation Forum. Springer International Publishing, Cham

Willcocks L (2020) Robo-Apocalypse cancelled? Reframing the automation and future of work debate. J. Inf. Technol.:1-17. https://doi.org/10.1177/0268396220925830

Yin RK (2018) Case study research and applications: Design and methods, 6th edn. SAGE, Los Angeles

Zhang C (2019) Intelligent Process Automation in Audit. J. Emerg. Technol. Account 16:69-88. https://doi.org/10.2308/jeta-52653 